\title{
Single-patient multiple crossover studies to determine the effectiveness of paracetamol in relieving pain suffered by patients with advanced cancer taking regular opioids: A pilot study
}

Palliative Medicine

I-3

(C) The Author(s) 2016

Reprints and permissions:

sagepub.co.uk/journalsPermissions.nav DOI: $10.1177 / 0269216316635012$ pmj.sagepub.com

(S)AGE

\author{
Jane Nikles', Geoffrey K Mitchell', Janet Hardy², Hugh Senior ${ }^{3}$, \\ Sue-Ann Carmont', Philip J Schluter, 4,5 , Rohan Vora ${ }^{6}$, \\ David Currow ${ }^{7}$ and Michael Yelland ${ }^{8}$
}

\begin{abstract}
What is already known about the topic?
- Paracetamol is a useful adjunct when used in combination with "weak opioids" for chronic pain in palliative care patients with advanced cancer; however, it is not certain that there is continuing benefit when used in conjunction with "strong" opioids.
\end{abstract}

What this paper adds?

- $\quad \mathrm{N}$-of-I trials allowed individual treatment decisions to be made for each participant: there was no added benefit for any of the participants, although no conclusion about the added benefit of paracetamol above regular opioids was possible for the group, due to insufficient numbers recruited.

Implications for practice, theory, or policy

- Paracetamol may not provide added benefit above regular opioids; this should be assessed on a case-by-case basis to justify the extra tablet load.

\section{Background and rationale}

Chronic pain is the most feared symptom in palliative care (PC) patients. While paracetamol is effective for mild to moderate pain and useful when used in combination with "weak opioids," it is not certain that there is sufficient continuing benefit when used in conjunction with "strong" opioids for moderate to severe pain to justify the additional medication burden.

Although there have been several studies describing opioid-sparing effects of paracetamol, there have been only a handful in patients with advanced cancer and pain despite strong opioids. A 2013 critical review concluded that the role of paracetamol in management of cancer pain remains controversial. ${ }^{1}$ We conducted a series of n-of- 1 trials to test the feasibility of using this methodology to obtain the per patient effectiveness of paracetamol in providing additional analgesia to regular opioids in people with advanced cancer experiencing moderate to severe pain.
IUniversity of Queensland Centre for Clinical Research, The University of Queensland, Herston, QLD, Australia

${ }^{2}$ Department of Palliative and Supportive Care, Mater Health Services, Brisbane, QLD, Australia

${ }^{3}$ College of Health, Massey University, Auckland, New Zealand

${ }^{4} \mathrm{~S} c h o o l$ of Health Sciences, University of Canterbury, Christchurch, New Zealand

${ }^{5}$ School of Nursing, Midwifery and Social Work, The University of Queensland, Brisbane, QLD, Australia

${ }^{6}$ Department of Palliative Care, Gold Coast Health Service District, Gold Coast, QLD, Australia

${ }^{7}$ Discipline Palliative and Supportive Services, Flinders University, Bedford Park, SA, Australia

${ }^{8}$ School of Medicine, Griffith Health Institute, Griffith University, Gold Coast, QLD, Australia

\section{Corresponding author:}

Jane Nikles, School of Medicine, University of Queensland Centre for Clinical Research, The University of Queensland, Herston, QLD 4029, Australia.

Email: uqjnikle@uq.edu.au 


\section{Methods}

\section{Design}

We conducted double-blind, placebo-controlled three-cycle multiple crossover n-of- 1 trials in individual patients. Each cycle was 6 days, comprising two 3-day treatment periods of slow release paracetamol $665 \mathrm{mg}$ tabs (GlaxoSmithKline; over-encapsulated), or identical placebo, two capsules three times per day. Data from the first day of each 3-day period were discarded. The order of drugs in each cycle was determined by a random allocation schedule with block size of four, set prior to commencement of recruitment. Allocation was blinded to clinician, investigator, and patient.

Patient-completed daily diaries recorded Brief Pain Inventory (BPI) scores. ${ }^{2}$ The trial result was compared to a predetermined clinically important change of 2.0 from baseline in BPI pain on average score over the last $24 \mathrm{~h} .{ }^{3}$ Individual patient reports were generated to allow consultation between participant and clinician to decide whether to continue paracetamol.

\section{Study population}

Patients with advanced cancer taking opioids for moderate to severe chronic pain, in Queensland, Australia, from May 2010 to September 2012.

Approvals were obtained from Institutional Review Boards (IRBs) of participating hospitals, and the University of Queensland. All patients provided written informed consent.

\section{Inclusion criteria}

(a) Aged $\geqslant 18$ years;

(b) Clinical diagnosis of chronic-cancer-related pain with BPI average pain score of $\geqslant 3$ over previous $24 \mathrm{~h}$;

(c) Taking regular dose of opioids (excluding codeine or tramadol) stable in $48 \mathrm{~h}$ prior;

(d) $\leqslant$ Three doses of stable dose of breakthrough opioid per day in $48 \mathrm{~h}$ prior;

(e) Stable dose of other regular pain medications for at least $48 \mathrm{~h}$ prior. Patients already on paracetamol had to stop paracetamol 3 days prior;

(f) No intervention that might alter pain levels during 2 weeks prior or plans to undergo such on study;

(g) Intact gastrointestinal tract.

\section{Exclusion criteria}

(a) Liver function (aspartate transaminase (AST) and alanine transaminase (ALT)) $>1.5 \times$ upper limit of normal, total bilirubin outside normal range;

(b) Paracetamol allergy; (c) Cognitive impairment;

(d) Life expectancy $<6$ weeks;

(e) Poor understanding of English.

\section{Results}

Overall, seven participants commenced, with three completing three cycles, one completing two cycles, one completing one cycle, and two not completing any cycles.

Individual patients' mean differences in BPI Pain on average between paracetamol and placebo ranged from 0.2 to 0.3 with no patient having an important positive or negative response. Addition of paracetamol gave no added benefit above placebo. For three patients, paracetamol provided small additional pain relief in secondary outcomes, but this was not clinically significant.

\section{Adverse events}

There were two grade 1 adverse events (irregular heart beat) and three grade 2 adverse events (constipation), unrelated to paracetamol. There were no reported serious adverse events.

\section{Discussion}

Taking paracetamol in addition to opioids did not outweigh the additional tablet burden for any of the patients.

We demonstrated the considerable advantage of participating in n-of- 1 trials over randomized controlled trials (RCTs), namely, participation gave useful evidence for every participant who completed at least one cycle.

Recruitment was more difficult than expected. Barriers to recruitment were not related to the n-of- 1 trial design, but to the strict inclusion criteria, including the need to have been on a stable opioid dose. Many potential participants had far advanced disease and pain control requiring frequent medication adjustments thereby excluding them from participating. The extent of this problem was not fully anticipated and resulted in only seven eligible participants being recruited. There were also other problematic issues including the time taken to obtain ethics and governance approval for each site. A decision was made not to proceed to a full trial in this patient population. For future research in this area, we recommend targeting community patients earlier in the cancer trajectory, with less stringent eligibility criteria.

\section{Acknowledgements}

The authors thank the patients and clinicians who participated. J.H., G.K.M., D.C., and J.N. conceived and participated in the design of the study. J.N. drafted the manuscript. P.J.S. provided 
statistical advice and data analysis. All authors contributed to and approved the final manuscript.

ANZCTR no: ACTRN12609000870257.

\section{Declaration of conflicting interests}

The author(s) declared no potential conflicts of interest with respect to the research, authorship, and/or publication of this article.

\section{Funding}

The authors thank the NHMRC for funding (519791) and for providing an NHMRC post-doctoral research fellowship 401780 for Jane Nikles.

\section{References}

1. Mercadante $\mathrm{S}$ and Giarratano A. The long and winding road of non steroidal antinflammatory drugs and paracetamol in cancer pain management: a critical review. Crit Rev Oncol Hematol 2013; 87(2): 140-145.

2. Cleeland CS. Pain assessment in cancer. In: Osoba D (ed.) Effect of cancer on quality of life. Boca Raton, FL: CRC Press, 1991, pp. 293-305.

3. Dworkin RH, Turk DC, Wyrwich KW, et al. Interpreting the clinical importance of treatment outcomes in chronic pain clinical trials: IMMPACT recommendations. J Pain 2008; 9: 105-121. 